\title{
The role of recombination in the emergence of a complex and dynamic HIV epidemic
}

\author{
Ming Zhang ${ }^{1,2^{*}}$, Brian Foley ${ }^{1}$, Anne-Kathrin Schultz ${ }^{3}$, Jennifer P Macke ${ }^{1}$, Ingo Bulla ${ }^{3}$, Mario Stanke ${ }^{3}$, \\ Burkhard Morgenstern ${ }^{3}$, Bette Korber ${ }^{1,4}$, Thomas Leitner ${ }^{{ }^{*}}$
}

\begin{abstract}
Background: Inter-subtype recombinants dominate the HIV epidemics in three geographical regions. To better understand the role of HIV recombinants in shaping the current HIV epidemic, we here present the results of a large-scale subtyping analysis of 9435 HIV-1 sequences that involve subtypes A, B, C, G, F and the epidemiologically important recombinants derived from three continents.

Results: The circulating recombinant form CRF02_AG, common in West Central Africa, appears to result from recombination events that occurred early in the divergence between subtypes $A$ and $G$, followed by additional recent recombination events that contribute to the breakpoint pattern defining the current recombinant lineage. This finding also corrects a recent claim that $\mathrm{G}$ is a recombinant and a descendant of CRF02, which was suggested to be a pure subtype. The BC and BF recombinants in China and South America, respectively, are derived from recent recombination between contemporary parental lineages. Shared breakpoints in South America BF recombinants indicate that the HIV-1 epidemics in Argentina and Brazil are not independent. Therefore, the contemporary HIV-1 epidemic has recombinant lineages of both ancient and more recent origins.

Conclusions: Taken together, we show that these recombinant lineages, which are highly prevalent in the current HIV epidemic, are a mixture of ancient and recent recombination. The HIV pandemic is moving towards having increasing complexity and higher prevalence of recombinant forms, sometimes existing as "families" of related forms. We find that the classification of some CRF designations need to be revised as a consequence of (1) an estimated $>5 \%$ error in the original subtype assignments deposited in the Los Alamos sequence database; (2) an increasing number of CRFs are defined while they do not readily fit into groupings for molecular epidemiology and vaccine design; and (3) a dynamic HIV epidemic context.
\end{abstract}

\section{Background}

Retroviral recombination introduces rapid, large genetic alternations [1-3], and can repair genome damage [4,5]. Recombination is a major force in HIV evolution, occurring at an estimated rate of at least 2.8 crossovers per genome per cycle [6]. Recently the effective recombination rate, i.e., the product of super-infection and crossovers, was estimated to be on a similar frequency as the nucleotide substitution rate within patients $\left(1.4 \times 10^{-5}\right.$ recombinations per site and generation) [7]. Recombination between HIV-1 subtypes may result in establishing epidemiologically important founder strains. Recombinant lineages can contribute to secondary recombination

\footnotetext{
* Correspondence: mingzh@lanl.gov; tkl@lanl.gov

'Theoretical Biology \& Biophysics, Los Alamos National Laboratory, Los Alamos, NM 87545, USA
}

events, leaving traces of ever more complex diversity patterns and confounding classical phylogenetics [8]. Within a single host, recombination may produce variants resistant to HIV-1 specific drugs and immune pressure [9-12].

At least $20 \%$ of HIV-1 isolates sequenced worldwide are inter-subtype recombinants [13-16]. These recombinants are classified into two categories, CRFs (circulating recombinant forms) and URFs (unique recombinant forms), referring to recombinants that have established recurrent and transmitted forms in populations, and to those only identified in one individual, respectively [17]. Currently, more than 40 CRFs and 100 URFs have been identified worldwide http://www.hiv.lanl.gov. Globally, these numbers are increasing as a result of multiple subtypes (and recombinants) in local epidemics, thus

\section{() Biomed Central}


providing the biological context for inter-subtype recombination. The number of detected recombinants is also increasing due to improved technology allowing rapid large-scale genome sequencing and the availability of more advanced recombination detection software.

It is estimated that CRF02_AG, a CRF derived from subtype A and G, has caused at least 9 million infections worldwide [18]. First identified in Nigeria in 1994 [19], it is the most prevalent strain in West and West Central Africa. In Cameroon, where the original HIV-1 M group zoonotic transmissions are believed to have taken place $[20,21]$, CRF02 was already prevalent in the early 1990s [22], and it is currently the dominant lineage in this part of the world $[20,23]$. It is possible that CRF02's high prevalence in Africa is explained by its long presence in the epidemic. Comparing the genetic diversity within CRF02 to that occurring within pure subtypes, Carr et al. suggested that CRF02 may be as old as the pure subtypes [24]. A recent study proposed the idea that CRF02_AG was a parent of subtype G [25], rather than subtype A and G being parental strains of CRF02.

CRF07_BC and CRF08_BC are the most common BC recombinants. CRF07 was first identified in the Xinjiang province of China in 1997 [18,21,26,27], and it is believed to have migrated to Xinjiang along a northern drug trafficking route $[26,28]$. CRF08 is a predominant subtype among intravenous drug users (IDUs) in Guangxi and the east part of the Yunnan province in China $[26,29]$. Both CRFs presumably originated in Yunnan where subtypes $\mathrm{B}$ and $\mathrm{C}$ were co-circulating in the early 1990s [30-33], or in Myanmar and then imported from there into China [34-37]. It has not been established whether other $\mathrm{BC}$ recombinants in Myanmar and China are epidemiologically linked to the CRF07 and CRF08 HIV-1 epidemic in Southern China [38,39].

BF recombinants in South America are dominated by a large number of recombinants with unique breakpoint patterns, URFs http://www.hiv.lanl.gov, geography page. The BF epidemic in this region is characterized by two genetic centers. One is represented by CRF12_BF and related genomes that are more frequently found in Argentina; and the other by CRF28_BF, CRF29_BF and a collection of BF URFs that have been found in Brazil [40]. The origin of BF recombinants in South America is not clear, but it appears that at least one of the main introductory routes of HIV-1 into South America was through Brazil [41].

Accurate virus genotyping and recombination identification techniques are important for many reasons, including epidemiological tracking, targeting vaccines to regional epidemics, understanding the evolutionary trajectory of the virus, and defining potential phenotypic differences in different subtypes or inter-subtype recombinants [42]. Here we report results from a large-scale subtyping study of 9435 sequences that includes subtypes A, B, C, G, F, and CRFs and URFs exclusively composed of subtypes $\mathrm{A}$ and $\mathrm{G}$, or $\mathrm{B}$ and $\mathrm{C}$, or B and $\mathrm{F}$. These sequences include all circulating recombinant forms dominating three epidemically important regions: West and West Central Africa, southern China, and South America. A series of detailed analyses were performed to ensure genotyping quality. Therefore, our analyses can provide a more comprehensive image of the current HIV epidemics in these three geographic regions. We demonstrate strong evidence that the recombinant lineages that are highly prevalent in the current HIV epidemic are a mixture of ancient and recent recombinant lineages. The dynamic HIV epidemic is moving toward having increasing complexity and higher prevalence of recombinant forms. Finally we suggest that a revision of some CRFs may be needed.

\section{Results}

\section{Genotyping results and comparisons to the original subtype assignments suggest that a revision of some CRF designations may be needed}

In total, we genotyped 9435 near full-length and sequence fragments obtained from the Los Alamos HIV sequence database and compared our results to the subtype assignments derived from the original literature (Table 1). Overall, $4.9 \%$ of the subtype assignments were inconsistent. The number of inconsistent assignments were unevenly distributed among sequence lengths such that shorter fragments more often than near full-length sequences disagreed: Among BC recombinants, 59.6\% of the sequence fragments were assigned differently in our results as compared to the original author assignments (Table 1, BC column). This difference is, however, not as dramatic as it may seem. For example, all literatureassigned CRF08 sequence fragments were assigned as pure subtypes in our results - in one case subtype B and in the rest subtype $\mathrm{C}$. Given that it is difficult to resolve the subtype in un-sequenced regions outside a sequence fragment, it becomes a philosophical nomenclature question of which assignment is best for sequence fragments embedded in a genomic region that is spanned by just one subtype constituting the locally prevalent CRF, i.e., to assign a sequence fragment with "CRF08", "C" or "B". When the HIV nomenclature procedures were first outlined [17], for the sake of consistency, there was a decision to use the subtype designation when a fragment was too short to span known breakpoints for CRFs. Thus the convention we use assigns the sequences based on the available information, e.g., a $\mathrm{C}$ fragment should be assigned as " $\mathrm{C}$ " even if it is suggested that CRF08 is known to be common in the geographic region where the sequence was isolated and even if the $C$ is closer to the $C$ in CRF08 rather than to 
Table 1 Comparison of subtype assignments (jpHMM results versus current database assignment that is based on the original literature)

\begin{tabular}{|c|c|c|c|c|c|c|c|c|c|c|c|c|c|c|c|}
\hline \multirow{2}{*}{\multicolumn{2}{|c|}{ Num of sequences }} & \multicolumn{5}{|c|}{ AG set } & \multicolumn{8}{|c|}{ BC set } & \\
\hline & & \multicolumn{4}{|c|}{$\begin{array}{l}\text { Full length (world) } \\
\qquad N=140\end{array}$} & \multicolumn{5}{|c|}{$\begin{array}{l}\text { Full length (world) } \\
\qquad N=509\end{array}$} & \multicolumn{5}{|c|}{$\begin{array}{l}\text { Fragments (Asia) } \\
\mathrm{N}=4413\end{array}$} \\
\hline \multicolumn{2}{|l|}{ Database subtype } & A & G & 02 & AG & B & C & 07 & 08 & $\mathrm{BC}$ & B & C & 07 & 08 & BC \\
\hline \multicolumn{2}{|l|}{ Num of sequences } & 72 & 12 & 48 & 8 & 152 & 334 & 7 & 4 & 12 & 3133 & 1048 & 17 & 171 & 44 \\
\hline \multicolumn{2}{|l|}{ Num of problematic sequences ${ }^{1}$} & 1 & 0 & 2 & 0 & 15 & 12 & 0 & 0 & 3 & 0 & 0 & 0 & 0 & 0 \\
\hline \multicolumn{2}{|l|}{ Num of discordant sequences ${ }^{2}$} & 0 & 0 & 1 & 0 & 2 & 0 & 0 & 0 & 2 & 24 & 6 & 6 & 102 & 27 \\
\hline & & \multicolumn{14}{|c|}{ BF set } \\
\hline Num of sequences & & \multicolumn{7}{|c|}{$\begin{array}{l}\text { Full length (world) } \\
\qquad N=220\end{array}$} & \multicolumn{7}{|c|}{$\begin{array}{l}\text { Fragments (S. America) } \\
\qquad \mathrm{N}=4153\end{array}$} \\
\hline Database subtype & B & & $=$ & 12 & 17 & 28 & 29 & $\mathrm{BF}$ & B & $\mathrm{F}$ & 12 & 17 & 28 & 29 & $\mathrm{BF}$ \\
\hline Num of sequences & 152 & & 2 & 11 & 2 & 3 & 4 & 36 & 3070 & 242 & 261 & 0 & 0 & 0 & 580 \\
\hline Num of problematic sequences ${ }^{1}$ & 15 & & ) & 0 & 0 & 0 & 0 & 0 & 0 & 0 & 0 & 0 & 0 & 0 & 0 \\
\hline Num of discordant sequences ${ }^{2}$ & 2 & & 2 & 6 & 2 & 1 & 1 & 1 & 74 & 19 & 31 & 0 & 0 & 0 & 107 \\
\hline
\end{tabular}

1. Problematic sequences are those that could not be unequivocally assigned. They meet one of the following criteria: 1) Contain an unusually high content of IUPAC code $\mathrm{N}$ (defined as $>100$ continuous $\mathrm{Ns}$, or $>7 \% \mathrm{~N}$ for sequences of length $<1000 \mathrm{nt}$, or $>5 \% \mathrm{~N}$ for sequences of length $1000-2999$, or $>3 \% \mathrm{~N}$ for sequences of length 3000 or above); 2) Contain an artifactual deletion of $>100 \mathrm{nt}$.

2. Classification of the sequences was compared between the database assignments (of which the majority were extracted from the literature) and the jpHMM predictions.

a pure $C$ (note also that this distinction often cannot be made with confidence). Finally, unless the whole genome is sequenced one cannot know what the classification is in uninvestigated regions. Thus, in agreement with the original HIV nomenclature proposal we have assigned fragments to their closest subtypes (or CRF) but not guessed what the rest of the genome is.

Next, all near full-length AG, BC, and BF recombinants were grouped into common groups if the sequences had similar genomic structures and breakpoints (Figs. 1, 2, and 3). Our results suggested that revisions of some CRF designations may be needed. For instance, some database-assigned BF CRF sequences in this analysis appear to be unique BF URFs with atypical breakpoints (Fig. 3). In case of CRF17, two previous sequences (accession number: AY037275 and AY037277) were assigned as CRF17 prototype sequences. They were, however, epidemiologically linked [43]. Another 7 sequences of CRF17 (mostly unpublished) have now been made available. These sequences consist of related, but not identical, recombinant forms that could be described as a "family" of recombinants (see further discussion on this topic).

The CRF and URF sequences described below refer to the sequences confirmed by our jpHMM genotyping results.

\section{CRF02 is a recombinant lineage with both early and more recent recombination events involving subtypes $A 1$ and $G$}

To examine the evolutionary relationships among recombinants that are exclusively composed of subtypes
A and G, as well as their relationships with all sequences of pure subtypes A and G, we performed phylogenetic analyses in eight sub-regions (Fig. 4, Regions I-VIII) delimited by the shared breakpoints of most full-length AG sequences depicted in Figure 1. IBNG is considered a prototype strain of CRF02, and was found representative of the most common AG-lineage (Group 1, Fig. 1). Other sequences, however, did not cluster with the same subtype as IBNG in all studied genomic regions, indicating subsequent secondary recombination events with other $A$ and $G$ viruses. Interestingly, some genomic regions suggest that CRF02 is an old recombinant derived from representatives of subtypes $A$ and $G$ that are similar to the most recent common ancestor of the two clades. There, the CRF02 clade is a sibling lineage to contemporary subtype A and G sequences, branching nearest to, but outside of, the clade based on more current sequences (Fig. 4. Sibling of A in Regions I, III and sibling of G in Region II). The topologies of the trees also suggested that the current CRF02 has undergone multiple recombination events, and some genomic regions of the first generation of CRF02 sequences were replaced by more recent sequences (Fig. 4. CRF02 is a descendent lineage of A in Regions V, VI, and a descendent lineage of $\mathrm{G}$ in Region VII). To assess whether sibling and descendent phylogenetic classifications indicate older and more recent fragments, respectively, we analyzed the correlation between sampling time point and the height of taxa from its subtype most recent common ancestor (sMRCA). The largest subtype $G$ fragment (Region II) was sampled in 1991-2002 ( $\mathrm{N}=39$ taxa) and showed a correlation of 


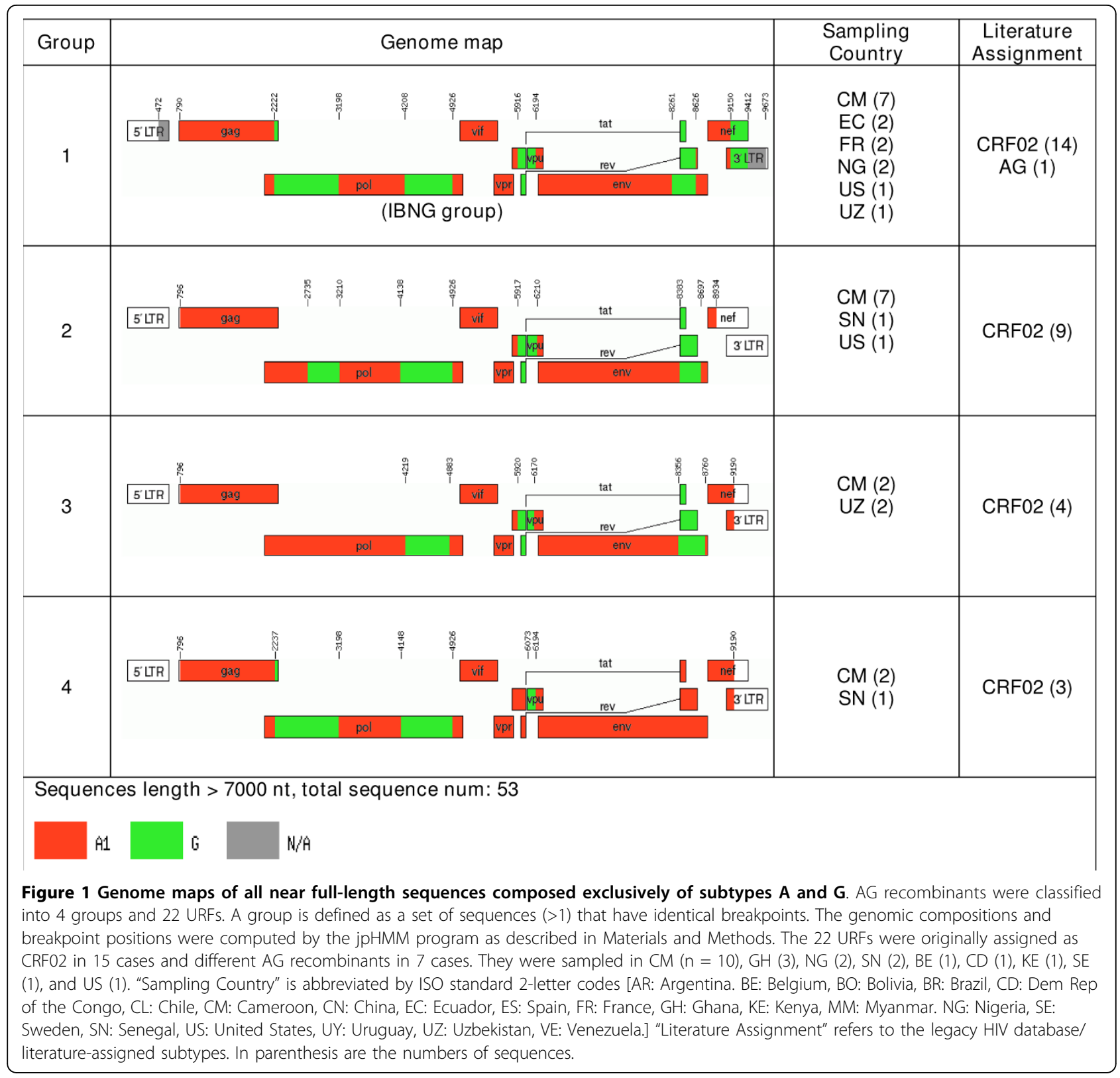

$\mathrm{R}=0.41$ between sampling time and tip height from its sMRCA ( $<<0.01$, F-test, linear regression). Likewise, the largest subtype A1 fragment (Region VI) which was sampled in 1985-2003 ( $\mathrm{N}=102$ taxa) had $\mathrm{R}=0.50$ $(\mathrm{P}<0.01, \mathrm{~F}$-test, linear regression). Note that the correlation coefficient $(\mathrm{R})$ is not dependent on the molecular clock being a constant rate clock, only that branches get longer with time; the P value does however depend on a linear trend estimation. Thus, our phylogenetic assignments of "old" and "new" are supported by the correlation between sampling time and growth of tip height from the respective sMRCA. The alignment quality was fairly even in terms of gap counts and the genetic diversity followed expected gene patterns (Additional file 1, Fig S1).

In agreement with our results, such second generation recombinants have been noted by others to be common [44]. Of particular interest, a recent argument, based on an analysis of Region IV suggests that CRF02 is a pure subtype and is a parent of the contemporary G clade which is the recombinant. This is in contrast to the current HIV nomenclature which suggests that the G clade is the parent and CRF02 the recombinant [25]. To clarify the confusing but critical argument, we investigated all CRF02 and G sequences derived from the Los Alamos HIV sequence database. While our tree 


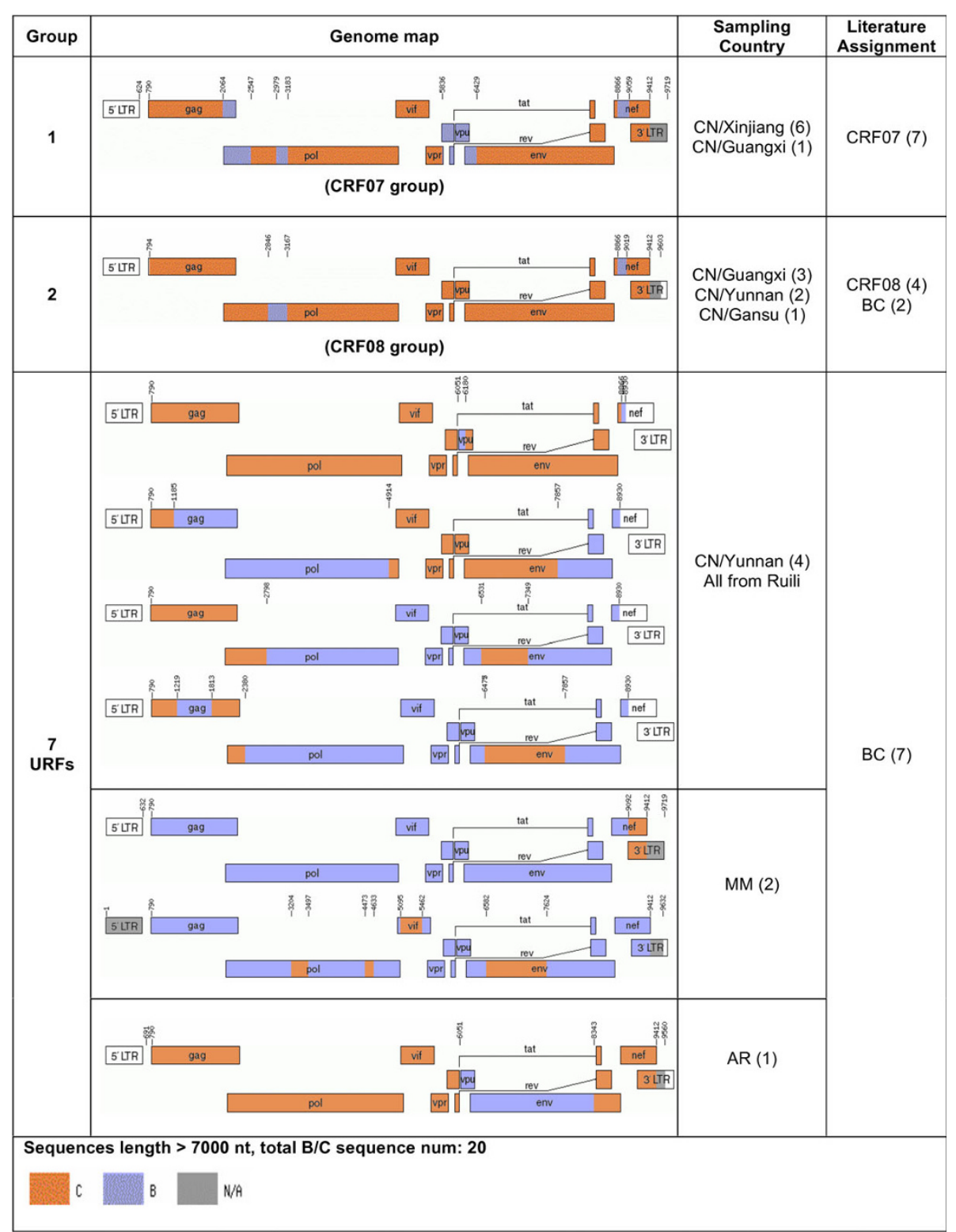

Figure 2 Genome maps of all near full-length sequences composed exclusively of subtypes B and C. BC recombinants were classified into 2 groups and 7 URFs. Group definitions and country codes are as in Fig 1.

suggested that CRF02 was inside the G clade in Region IV, there was no bootstrap support for this classification. Importantly, besides Region IV, the rest of the genome fragments (both A1 and G) had better bootstrap support and clearly indicated that $\mathrm{G}$ is a subtype and CRF02 a recombinant (Fig 4). Furthermore, a RIP analysis attempting to resolve the origin of Region IV (and others) showed that CRF02 was closer to a G maximum likelihood-inferred ancestor (G.anc) than to a G consensus of contemporary sequences (G.con) (CRF02 to G. anc $=0.0178$ substitutions $/$ site, and CRF02 to G.con $=$ 0.0218 substitutions/site) (Fig 4B). The likelihood was $\mathrm{p}<10^{-8}$ that G.anc and G.con were the same $(2 \Delta \operatorname{lnL}=$ 34.5 , general-time-reversible model with 9 site rates), but there were only two positions that differed in Region IV and thus this result should be interpreted with caution. For instance, the underlying model parameters could change if new sequences were included in the inference, potentially changing the state probabilities and the site likelihoods. Nevertheless, for Region IV, at this point the difference between G.anc and G.con are significant, CRF02 was found overall closer to G.anc, and at the two positions G.anc and G.con differed CRF02 was identical to G.anc, all together suggesting a 


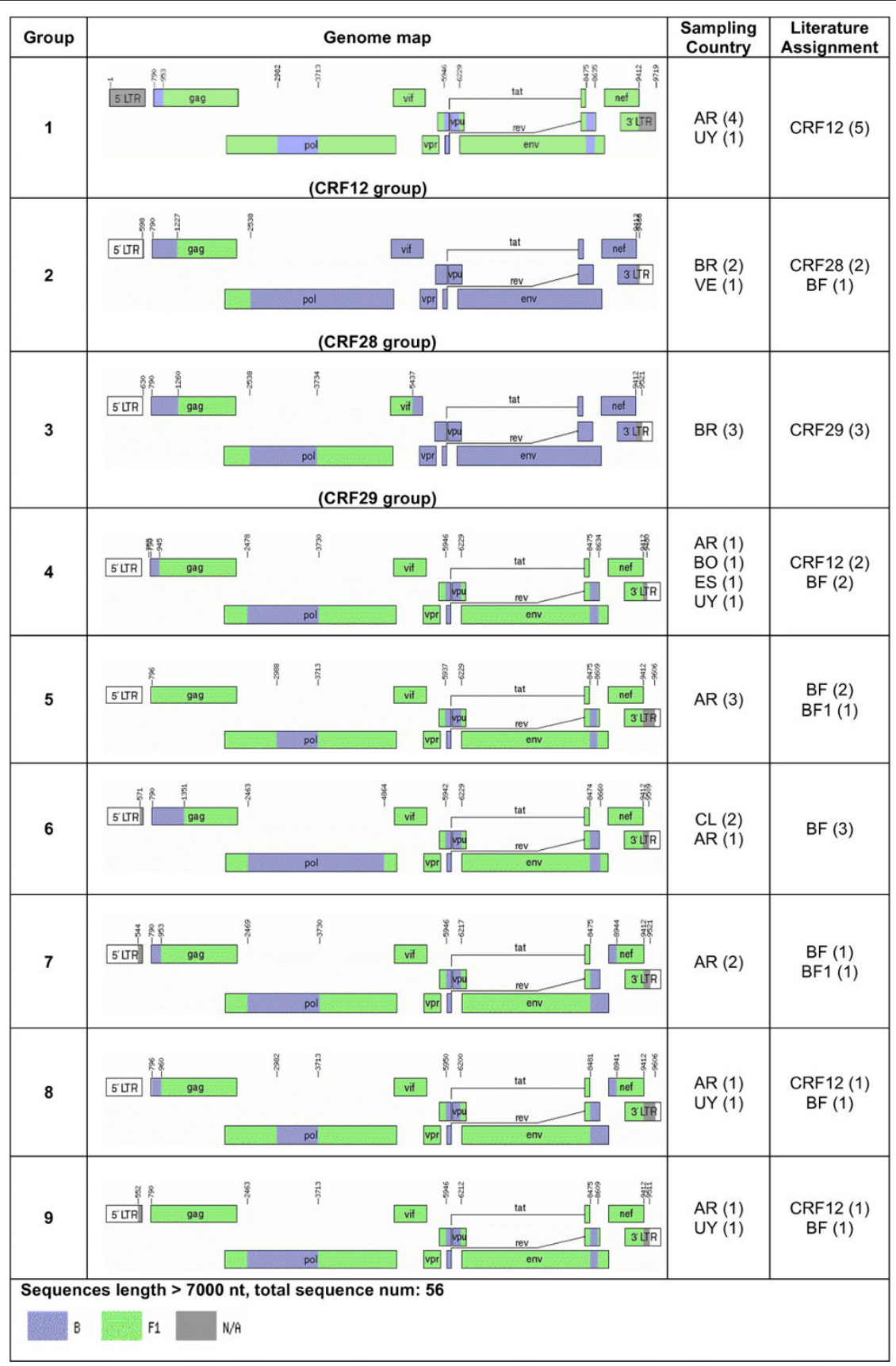

Figure 3 Genome maps of all near full-length sequences composed exclusively of subtypes B and F. BF recombinants were classified into 9 groups and 29 URFs. Group definitions and country codes are as in Fig 1. The 29 URFs were originally assigned as CRF $12(n=2)$, CRF17 (2), CRF28 (1), CRF29 (1), and different BF recombinants in 23 cases. They were sampled in BR ( $n=18), A R(9), C L(1)$, and ES (1).

more ancient origin of CRF02 Region IV. Also note that the RIP analysis showed that Region IV has the least power to resolve the phylogenetic classification of the CRF02 genome, because this region has the smallest amount of divergence (Fig 4B). This also explains the poor bootstrap support in Region IV tree. Further, although the sequences are highly similar, the maximum likelihood estimates of ancestral sequences of clades A and G should reflect better the ancestral state of the clade, incorporating phylogenetic information from the full $M$ group tree, while the consensus sequences derived from contemporary A and G isolates slightly favors contemporary forms. Thus, the RIP analysis further supported the tree results that some sections of the CRF02 genome may have involved old recombination events from a time when the clades were beginning 


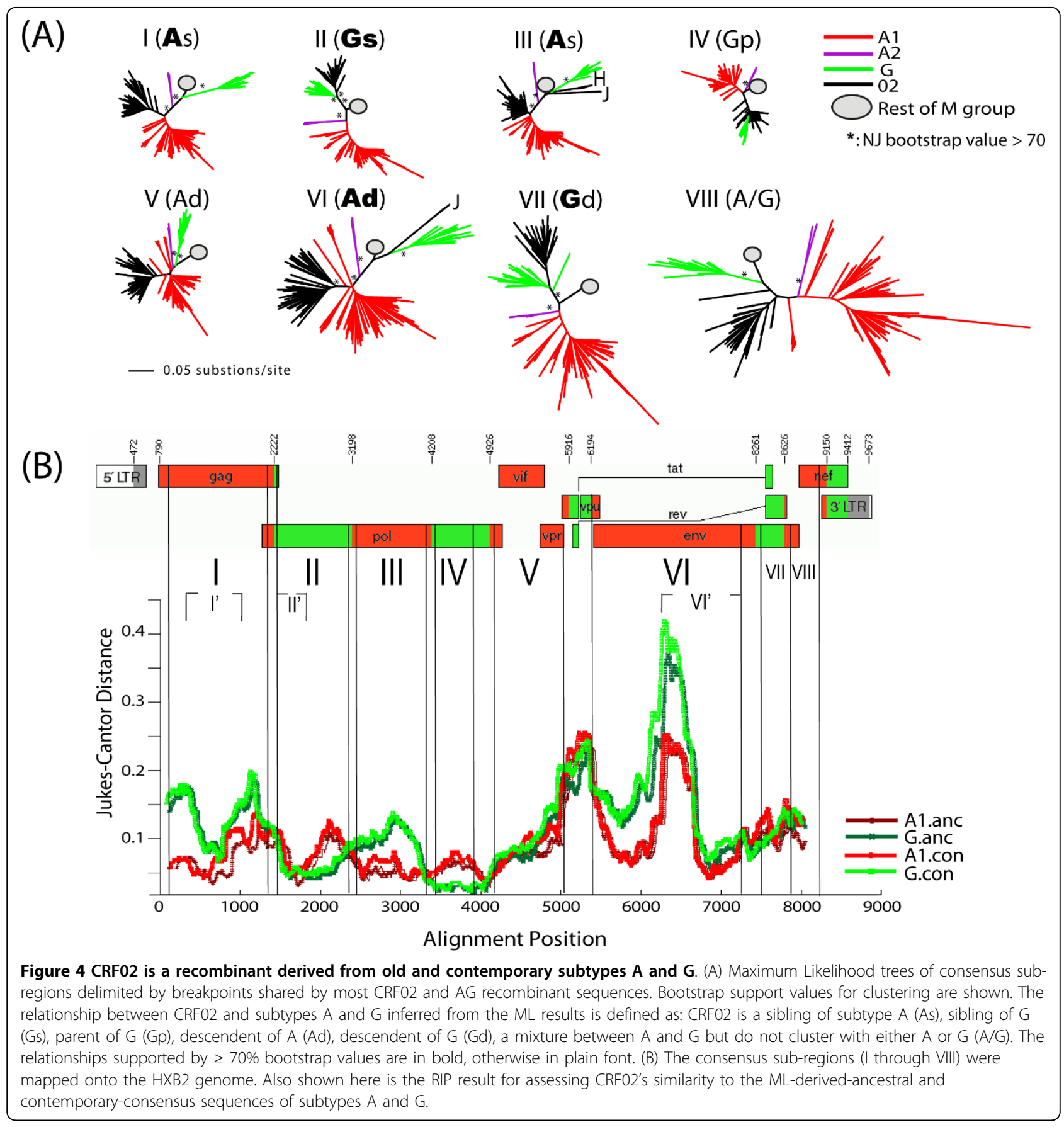

to diverge, and that some other regions were more likely to have involved more recent subtype A and G sequences. To avoid potential problems with the uncertainty of breakpoint locations, we also phylogenetically analyzed smaller sub-regions of the larger regions (I', II', and $\mathrm{VI}^{\prime}$ ) and found consistent results with the presented larger region analyses. In conclusion, taken all regions of the CRF02 genome into account, our analyses show that CRF02 is a recombinant of both ancient and more recent $\mathrm{A}$ and $\mathrm{G}$ parents.
The Chinese BC-recombinant epidemic was formed locally with limited contacts with most other Asian countries

To characterize the relationships of $\mathrm{BC}$ recombinants from China, Asia, and worldwide, we first investigated the relationship between CRF07 and CRF08. Full-length sequences classified as CRF07, CRF08, or BC were grouped according to their breakpoint structures (Fig. 2 ), and ML trees were constructed for sub-regions delimited by all CRF07 and CRF08 sequences (Fig. 5). While most of the examined sub-regions showed a 


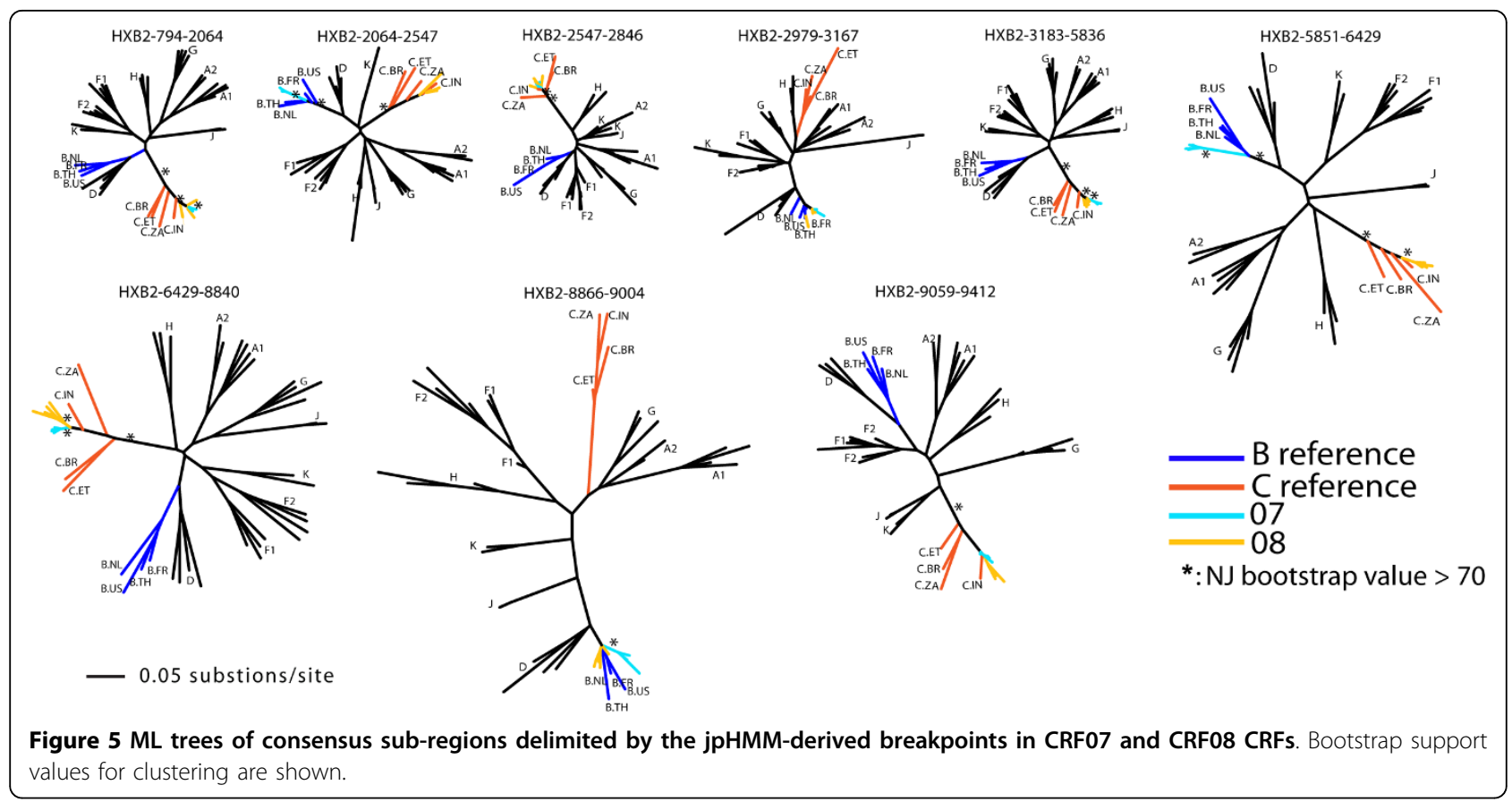

sibling relationship between CRF07 and CRF08, two sub-regions (HXB2 positions 794-2064 and 2547-2846) suggested that, at least in these sub-regions, CRF08 may be the parent of CRF07 because CRF07 sequences were clustered inside the CRF08 clade (bootstrap support $\geq$ 70\%). Further, CRF07 and CRF08 were derived from multiple recombination events, as indicated by unequal breakpoint frequencies in CRF07 and CRF08 (Fig. 6, top panels). The breakpoint at HXB2 position 8866 was consistent among CRF07, CRF08, and subsequent recombinants, and thus was likely to be introduced into CRF07 and CRF08 through a common ancestor.

To investigate $\mathrm{BC}$ recombinants from China and China's neighboring countries, phylogenetic analyses were performed on consensus sub-regions delimited by most near-full-length $\mathrm{BC}$ recombinants shown in Figure 2. There was a close relationship between Yunnan B and Myanmar B (data not shown). Sequences from these two geographic regions are very limited (6 BC sequences from Yunnan and 2 from Myanmar), therefore we cannot deduce the direction of the epidemic movement between Yunnan and Myanmar.

Finally, the influence of worldwide $\mathrm{B}$ and $\mathrm{C}$ epidemics on the Chinese $\mathrm{BC}$ recombinants was analyzed. As described in the Materials and Methods, the global set of subtype $B$ and $C$ sequences was retrieved from the HIV database in the genomic regions that had the longest subtype $\mathrm{B}$ and the longest $\mathrm{C}$ sub-regions shared by all CRF07, CRF08, and most near full-length $B C$ recombinants. In the subtype B sub-region tree, sequences from China appeared to be a local epidemic only involving neighboring countries Thailand and Myanmar (Additional file $1 \mathrm{Fig} . \mathrm{S} 2 \mathrm{~A}$ ); this occurred possibly through drug trafficking routes $[26,28]$. Other Asian countries, for instance, Korea, Japan, and Thailand, appeared to have greater subtype B diversity, which may be explained by more frequent contacts with each other and with the rest of the world. Finally, South American subtype B seems to have had multiple HIV introductions from Europe and North America. The result of the subtype $C$ sub-region tree also suggested that China $C$ is a mostly local epidemic, with some influx of subtype $\mathrm{C}$ from India, but not Africa as India has (Additional file 1, Fig. S2B). Finally, the dominant South American C epidemic appears to have derived from a single introduction from Africa $([45,46]$ and Additional file 1, Fig. S2).

\section{Contemporary Argentinean and Brazilian HIV epidemics are not independent}

Our study did not show any association between risk factors and BF CRF groups (Fig. 3). In the breakpoint frequency analyses of full-length BF sequences (Figure 6, BF panels) and BF sequence fragments (Additional file 1, Fig. S3) all identified BF breakpoints were found in more than one country in South America, and occasionally in countries from other continents. This suggests that, although the South American HIV epidemic is represented by two distinctive epicenters, the BF epidemic has moved back and forth between Argentina and Brazil. Indeed, the BF recombinant sequence fragments carry all the information that fills the gap in the 


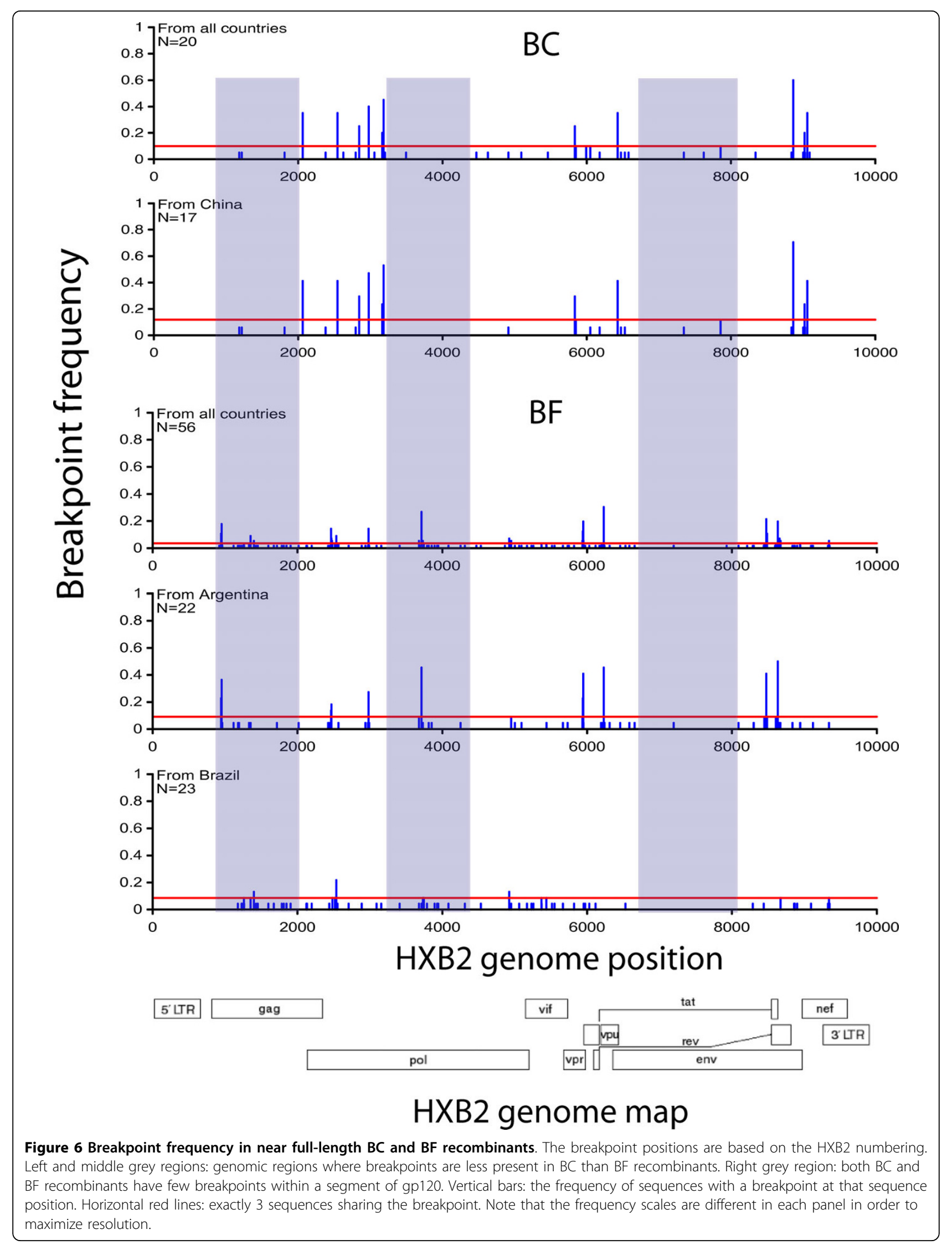


full-genome sequences from Argentina and Brazil such that all genomic regions of $B$ and $F$ can be found in either country. We also found that sequence V62 (accession number AY536236), which has an epidemiological linkage to the Argentinean epidemic [47], had the same genomic structure and breakpoints as CRF28, which was first described in Brazil. In all, the HIV epidemics in Argentina and Brazil are not independent.

We did not find evidence that Argentinean B and F were derived from Brazil, as previously suggested $[47,48]$. The result of the phylogenetic analyses, which agreed with previous publications $[40,49,50]$ and thus not shown here, demonstrated that $\mathrm{B}$ and $\mathrm{F}$ fragments of the jpHMM-confirmed CRF12, CRF28, and CRF29 were inter-mingled, and therefore could not support a single direction of HIV-1 flow. Also, as already mentioned, we found that Argentinean $B$ and $F$ sequence fragments in the HIV database can cover a full HIV-1 genome of each subtype, meaning that there was a potential to form any BF recombinants in Argentina and that there was no need to assume that already-recombined genomes came from Brazil. In addition, a recently identified near full-length Argentinean pure F sequence, ARE933 (accession number DQ189088), was found to be closer to Argentinean BF than were any other $\mathrm{F}$ strains [41,51]. The most likely scenario is that there were HIV-1 transmissions in both directions, with recombination of circulating strains in all countries involved.

\section{Discussion}

The geographic distribution of subtypes and recombinant lineages in any epidemic, influenced by local epidemiological factors, is dynamic and difficult to resolve. Here we present a large-scale subtyping re-analysis of $9435 \mathrm{HIV}-1$ sequences that involve subtypes A, B, C, G, $\mathrm{F}$, and their important CRFs in three different epidemiological settings that together have significantly shaped today's global HIV epidemic. Our comprehensive analyses demonstrate strong evidence that the contemporary HIV-1 epidemic is a mixture of recombinants that had an origin in the early HIV epidemic, likely before the subtypes were distinctively separated, while others are of more recent origin, and that shared breakpoints can be used for tracking patterns in the epidemic.

We found that CRF02 is a recombinant more complex than previously described. Its old origin, as well as the subsequent recombination events that occurred prior to the establishment of the contemporary CRF02 lineage, can easily confound the analysis of CRF02. Among the $\mathrm{BC}$ recombinants we found that the $\mathrm{BC}$ epidemic in China is unique compared to most other Asian countries; further, CRF07 and CRF08 were recently introduced to the epidemic, but both have undergone multiple recombination events. The study of BF recombinants in South Africa suggests that the HIV-1 epidemics in Argentina and Brazil are not independent.

The existence of early lineages in the current HIV-1 epidemic imposes a great challenge in detecting some recombinant sequences. Figure 7 shows a cartoon describing some of the difficulties described in this paper (e.g. CRF02) and also some effects of extinct (e.g. subtype "E") and undiscovered lineages. In addition to recombination effects, co-evolution of some sequence positions, for example due to fitness constrains and HLA-imposed immune pressure, gives rise to distinct but potentially convergent patterns of immune escape that can also confound recombination analyses by introducing homoplasy. Sometimes the history of old lineages can be recovered by extrapolating backward from surviving viruses (like subtype E [52,53]), while some lineages presumably can never be found (like lineage " $X$ " in Fig. 7). In this context, it is likely that [some of] the current pure subtypes are actually recombinants that were formed a long time ago, but because the "pure" parental lineages have been lost, we cannot trace their origin. Thus the current subtype nomenclature does not rest on the assumption that currently defined "pure" subtypes are not consequences of earlier recombination events, but rather indicates that these subtypes can be used as good background references in studying the current HIV-1 epidemic, and that the "pure" subtypes' relative genetic relatedness can provide a basis for studying and understanding the immunological consequences of diversity for vaccine design. Unfortunately, almost all existing genotyping tools are not well designed to infer old recombination events or for those that involve unknown parents.

The dynamic HIV-1 epidemic seems to have moved toward to have more complex recombinants. However the driving force may be different in different epidemiological settings. In Africa where the HIV epidemic is predominantly driven by heterosexual transmissions, the ancient history of CRF02 as described in this paper, together with its high replicative capacity $[54,55]$ and its high prevalence [56], make CRF02 an active participant in generating more and new complex recombinants, for instance, the newly identified CRF36_cpx [57]. BC recombinants in China will likely also continue to evolve. Super-infection of CRF07 and CRF08 viruses [28], as well as continuous influx of $B$ and $C$ into Yunnan from China's surrounding countries [58,59], contributes greatly to the emergence of new $\mathrm{BC}$ recombinants, notably BC URFs. Another important driving force of $\mathrm{BC}$ evolution in China is the rapid transition in the HIV-1 epidemic in some geographical regions. In Yunnan alone, subtype B was found to be the dominant subtype in the late 1980s, but it was soon replaced by 


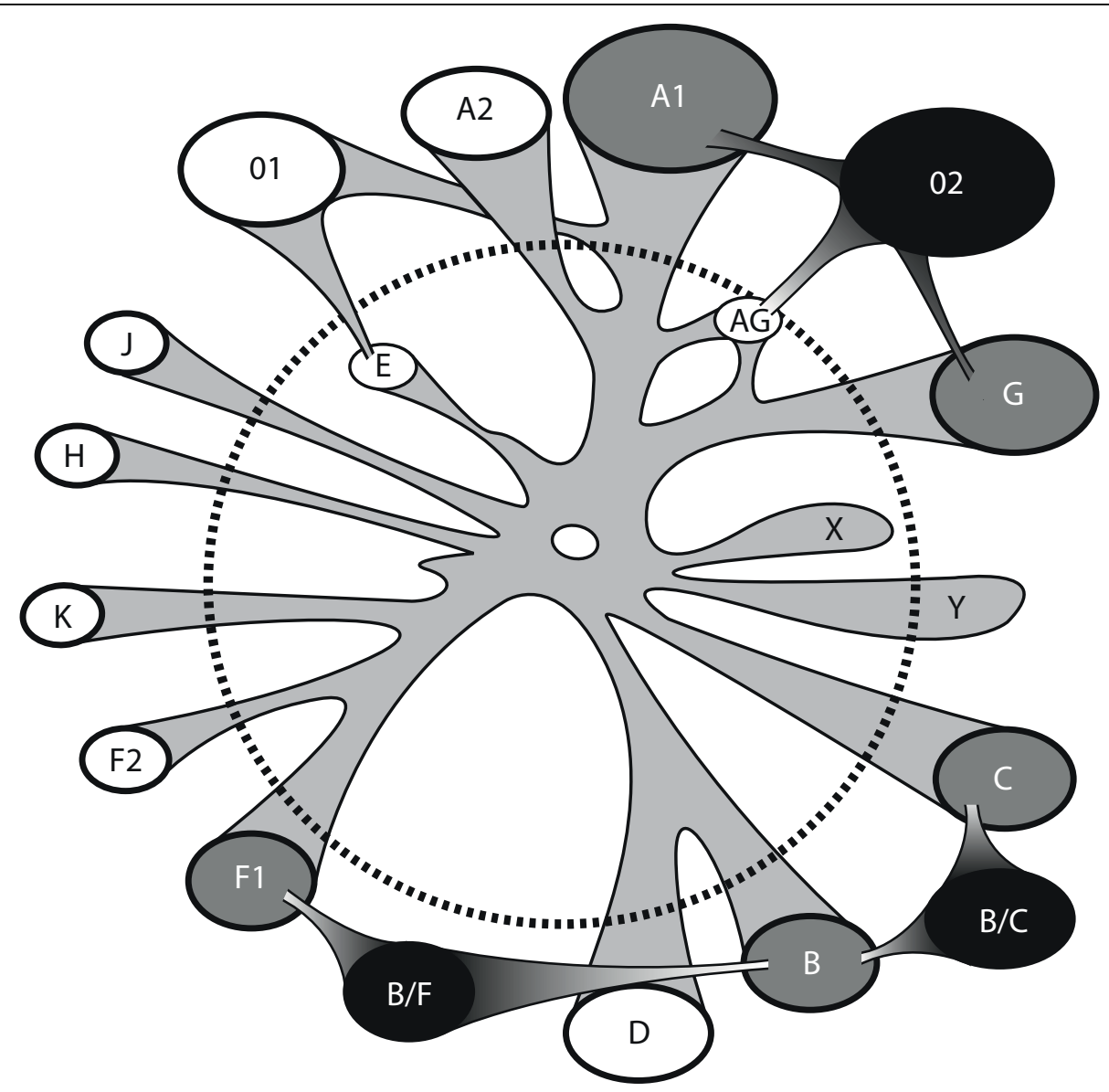

Figure 7 The current HIV-1 epidemic is a mixture of old and contemporary lineages. The dashed circle differentiates old and contemporary sequences. Inside the circle, the old sequences, such as the subtype $E$ clade, may no longer exist in the current epidemic. We can only infer the ancient presence of subtype $E$ based on CRF01_AE, a recombinant between subtype $A$ and $E$. " $X$ " represents a hypothetical extinct strain, "Y" represents a hypothetical old strain that is still circulating in the current epidemic, but hasn't been identified. CRF02 is an old recombinant derived from both old and contemporary subtype A and G. BF recombinants in South America and BC in China are new, as their parents are contemporary sequences. The black blobs (recombinants) and grey blobs ("pure" subtypes) are clades investigated in this paper, and white blobs are other HIV-1 clades.

Thai B; in 1992, subtype C was found in this region, thus Thai B and C co-circulated; in 1994, CRF01 was identified in Yunnan; in 2000 and 2001, subtype C was not detected among IDU samples in the same region $[28,30,37,58]$. While some of these transitions in the regional prevalence might have been a consequence of sampling biases, they still suggest complex patterns of epidemic dynamics. BF recombinants in South America are possibly moving toward having more URFs. A recent Bayesian hierarchical analysis also indicated extensive ongoing recombination among CRF12 viruses [60]. The long circulation record of subtypes B and F in South America $[43,61,62]$, and the tight HIV-1 transmission networks with high incidence rates found in some South American geographical regions may favor an elevated number of dual- and super-infections [48]. A possible outcome of this dynamic pattern is that pure subtype $\mathrm{F}$ may disappear after being gradually diluted from the South American epidemic. Hence, social network structures and possibly viral factors dictate the molecular epidemiology of HIV-1 [63]; and tracking the genetic lineages and patterns in recombination breakpoints can shed light on such factors.

Current CRF nomenclature requires all sequences of one CRF to have identical or very similar breakpoints, and thus originate from a single lineage of a recombinant form. Such breakpoints may, however, be easily blurred by subsequent substitutions and by further recombination events, as in the CRF02 and CRF17 cases described above. Hence, the sequences defined in a CRF are merely snapshots of the dynamic changes in HIV-1 evolution. This may cause problems when "new" CRFs are identified, which may be related to existing CRFs and not fit into meaningful groupings used for 
molecular epidemiology or vaccine design. It is questionable if the continuous use of these soon to be unmanageable number of CRFs will be useful to the HIV research community. Hence, we propose to define sequence "families" that would contain recombinant sequences composed of the same subtypes, but the sequences' genomic structures and breakpoints may not be identical due to successive recombination events or our inability to accurately describe them. Sequences would belong to one family as long as they are closer to a defined central strain of that family than to any other family, including "pure" subtypes, like the examples shown in Figs. 1, 2, 3. Each family would be defined by a central sequetype and the radius of family members would depend on distances in a multi-dimensional sequence space. Membership to a family could be based on overall similarity and, if needed, be further assessed by multidimensional scaling. Using such a "family" concept makes it feasible to dynamically track HIV diversity and epidemiologically important families over evolutionary time, regardless of their precise phylogenetic history.

\section{Conclusions}

Our large-scale re-subtyping meta-study provides a comprehensive view of HIV recombinants in three epidemically important regions. The dynamic HIV epidemic is moving toward having increasing complexity and higher prevalence of recombinant forms and it has posed a great challenge in many aspects of the HIV/ AIDS epidemic. We suggest that a revision of some CRFs may be needed. As we continue to systematically re-subtype the rest of the sequences in the Los Alamos sequence database, it is likely that our results will shed light on the impact of evolving HIV recombinants. This will give better database search results and may thus affect vaccine design and molecular epidemiology studies.

\section{Methods}

\section{Sequences}

The following sequence sets were retrieved from the Los Alamos HIV sequence database (http://www.hiv.lanl.gov sequence database search interface). Set 1: All near fulllength sequences (>7000 nucleotides [nt]) of subtypes A, B, C, F, G, CRF02, CRF07, CRF08, CRF12, CRF17, CRF28, CRF29, and all URFs composed exclusively of subtypes $A$ and $G$, or B and $C$, or B and F. Set 2: Shorter HIV sequences $(300-7000 \mathrm{nt})$ that are BC recombinants from Asia and BF recombinants from South America. Set 3: After examining all full-length BC recombinant genomes, we defined the longest subtype $B$ segment (HXB2 positions 3497-4473) and the longest subtype $\mathrm{C}$ region (HXB2 positions 6582-7349). For additional $\mathrm{BC}$ analyses, sequences covering these two fragments were retrieved from the database for all geographic regions worldwide. To avoid redundancy and reduce issues related to non-independence of data points, only 1 sequence per patient was included in the analyses of the sequence fragments. The analysis method used for Set 3 sequences, however, is not applicable to the AG and BF sequences due to the difficulties in obtaining accurate breakpoints (which is the case of CRF02; its old origin leads to fuzzy breakpoints) and in getting big enough genome regions (this is the case of South American BF in which URFs outnumber CRFs).

To examine further whether the risk factors contribute to the spread of BF URFs in South America, the associated risk factor of the near full-length $\mathrm{BF}$ recombinant sequences from South America was also retrieved from the database.

All sequences were aligned with HIV-1 subtype reference sequences (http://www.hiv.lanl.gov sequence alignment page) using GeneCutter (http://www.hiv.lanl.gov GeneCutter page). Alignment quality was checked manually in BioEdit [64] to ensure that the alignments did not contain obvious problems and that they were correctly codon aligned.

\section{Recombination detection}

Our jumping profile hidden Markov model (jpHMM) program $[65,66]$ was used to analyze the subtype assignment of all sequences retrieved. In jpHMM, each HIV-1 subtype is represented by a profile hidden Markov model. All profile models are connected by empirical probabilities, allowing the detection of possible recombinants and related breakpoints by jumping from one profile to another. jpHMM performs best in predicting recombinants that involve subtypes that have had adequate sampling to build well-informed profiles, i.e., it is less effective for subtypes $\mathrm{H}, \mathrm{J}$, and $\mathrm{K}$, because so few full-length genome sequences are available $(\mathrm{N}=3,3$, and 2 , respectively). In the present study, jpHMM was used to detect the recombination patterns in recombinants that are composed exclusively of subtypes $\mathrm{A}$ and G, B and C, or B and F; each of these subtypes has enough data to form a good model of sequence variation. For the recombinants detected, jpHMM provides detailed information about subtype composition and well-resolved breakpoint locations. The jpHMM source code is available at the jpHMM Web interface http:// jphmm.gobics.de.

\section{Phylogenetic analyses}

The near full-length sequences were grouped together if the sequences had similar subtype composition and breakpoint patterns. Sub-genomic regions delimited by shared breakpoints in the majority of AG recombinants 
(including jpHMM-confirmed CRF02 and AG URFs) were further analyzed using phylogenetic inference, discriminating between parental, descendent and sibling relationships. Initial screens of regions involving thousands of taxa, for example the global collection of the largest identified $\mathrm{B}$ and $\mathrm{C}$ genomic sub-regions in all near full-length $\mathrm{BC}$ recombinants (Set 3 ), were performed using PAUP neighbor joining with an F84 model [67]. More refined resolution of $B$ and $C$ recombinants, and all other primary trees involving subregions delimited by shared jpHMM-confirmed breakpoints, were done using PhyML with a GTR-Gamma model, enabling very large datasets to be analyzed phylogenetically [68]. The statistical robustness and the reliability of the notable clustering patterns in the ML trees were further evaluated by non-parametric bootstrap analyses in PAUP (neighbor-joining, F84 model, 1000 replicates). A bootstrap value of $\geq 70 \%$ was considered significant for subtype clustering [69].

\section{Further analysis of CRF02 origin}

The Recombination Identification Program version 3 (http://www.hiv.lanl.gov RIP3 page) was used along with likelihood trees to examine the relationship between CRF02 and contemporary sequences relative to maximum likelihood inferred ancestor sequences of subtypes A and G: A CRF02 consensus sequence was analyzed against an alignment that included ML-inferred ancestral sequences [70] (M group, $\mathrm{A} 1$, and $\mathrm{G}$ ) and consensus sequences ( $M$ group, $\mathrm{A} 1$, and $\mathrm{G}$ ). The CRF02 consensus was constructed based on the CRF02 sequences sets confirmed by jpHMM and phylogenetic analysis. Other consensus and ancestral sequences were retrieved from the HIV sequence database alignment page. All consensus and ancestral sequences were aligned using GeneCutter, followed by manual editing.

\section{Breakpoint frequency calculations}

Systematic breakpoint frequency calculations were performed on the following three sequence alignments: near full-length $\mathrm{BC}$ and $\mathrm{BF}$ recombinant sequences; fragments of $\mathrm{BC}$ recombinant sequences from Asia; and fragments of BF recombinant sequences from South America. The sequence subtyping and recombination patterns were derived from jpHMM analyses. The breakpoint frequencies of all sequences in each alignment were calculated and plotted. In BC CRFs, $>95 \%$ of the breakpoints were located within $16 \mathrm{nt}$ from the breakpoint median. In BF CRFs, > 95\% of the breakpoints were located within $98 \mathrm{nt}$ from the breakpoint median. These two numbers (16 nt and $98 \mathrm{nt}$ ) were used as breakpoint confidence regions for subsequent analyses of $\mathrm{BC}$ and $\mathrm{BF}$ recombinants, respectively, to provide boundaries for defining shared breakpoints.
Additional file 1: Supplementary figures S1, S2, S3. Suppl. figure 1. Gap frequency and mean pairwise distance in the CRF02 alignment. Suppl. figure 2. The BC epidemic in China is unique compared to China's neighboring countries. Suppl. figure 3 . The breakpoint frequency of CRF12, CRF28, and CRF29 sequences.

\section{Acknowledgements}

We greatly thank to Dr. William Fisher for helpful discussions. This work was supported by an NIH-DOE interagency agreement (Y1-Al-8309).

\section{Author details}

${ }^{1}$ Theoretical Biology \& Biophysics, Los Alamos National Laboratory, Los Alamos, NM 87545, USA. ${ }^{2}$ Center for Nonlinear Studies, Los Alamos National Laboratory, Los Alamos, NM 87545, USA. ${ }^{3}$ Institut für Mikrobiologie und Genetik, Abteilung Bioinformatik, Goldschmidtstraße 1, 37077 Göttingen, Germany. ${ }^{4}$ The Santa Fe Institute, Santa Fe, NM 87501, USA.

\section{Authors' contributions}

$T L, M Z$ and $B K$ designed and carried out the genotyping procedure. $M Z, T L$, $B K, B F$ and $J M$ analyzed the data. AS, MZ, BM, TL, IB, MS, BK developed and evaluated the jpHMM software. MZ, TL and BK wrote the manuscript. All authors approved the final version of the manuscript.

\section{Competing interests}

The authors declare that they have no competing interests.

Received: 30 September 2009 Accepted: 23 March 2010

Published: 23 March 2010

\section{References}

1. Temin HM: Retrovirus variation and reverse transcription: abnormal strand transfers result in retrovirus genetic variation. Proc Natl Acad Sci USA 1993, 90:6900-6903.

2. Hu WS, Temin HM: Retroviral recombination and reverse transcription. Science 1990, 250:1227-1233.

3. Hu WS, Temin HM: Genetic consequences of packaging two RNA genomes in one retroviral particle: pseudodiploidy and high rate of genetic recombination. Proc Natl Acad Sci USA 1990, 87:1556-1560.

4. Clavel F, Hoggan MD, Willey RL, Strebel K, Martin MA, Repaske R: Genetic recombination of human immunodeficiency virus. J Virol 1989, 63:1455-1459.

5. Stahl FW: Genetic recombination. Sci Am 1987, 256:90-101.

6. Zhuang J, Jetzt AE, Sun $G, Y$ Y H H, Klarmann G, Ron Y, Preston BD, Dougherty JP: Human immunodeficiency virus type 1 recombination: rate, fidelity, and putative hot spots. J Virol 2002, 76:11273-11282.

7. Neher RA, Leitner T: Recombination rate and selection strength in HIV intra-patient evolution. PLoS Comput Biol 2010, 6:e1000660.

8. Konings FA, Haman GR, Xue Y, Urbanski MM, Hertzmark K, Nanfack A, Achkar JM, Burda ST, Nyambi PN: Genetic analysis of HIV-1 strains in rural eastern Cameroon indicates the evolution of second-generation recombinants to circulating recombinant forms. J Acquir Immune Defic Syndr 2006, 42:331-341.

9. Jung A, Maier R, Vartanian JP, Bocharov G, Jung V, Fischer U, Meese E, Wain-Hobson S, Meyerhans A: Multiply infected spleen cells in HIV patients. Nature 2002, 418:144.

10. Moutouh L, Corbeil J, Richman DD: Recombination leads to the rapid emergence of HIV-1 dually resistant mutants under selective drug pressure. Proc Natl Acad Sci USA 1996, 93:6106-6111.

11. Vijay NN, Vasantika, Ajmani R, Perelson AS, Dixit NM: Recombination increases human immunodeficiency virus fitness, but not necessarily diversity. J Gen Virol 2008, 89:1467-1477.

12. Nora T, Charpentier C, Tenaillon O, Hoede C, Clavel F, Hance AJ: Contribution of recombination to the evolution of human immunodeficiency viruses expressing resistance to antiretroviral treatment. J Virol 2007, 81:7620-7628.

13. Osmanov S, Pattou C, Walker N, Schwardlander B, Esparza J: Estimated global distribution and regional spread of HIV-1 genetic subtypes in the year 2000. J Acquir Immune Defic Syndr 2002, 29:184-190. 
14. Peeters M, Sharp PM: Genetic diversity of HIV-1: the moving target. Aids 2000, 14(Suppl 3):S129-140.

15. Peeters M, Toure-Kane C, Nkengasong JN: Genetic diversity of HIV in Africa: impact on diagnosis, treatment, vaccine development and trials. Aids 2003, 17:2547-2560.

16. Hemelaar J, Gouws E, Ghys PD, Osmanov S: Global and regional distribution of HIV-1 genetic subtypes and recombinants in 2004. AIDS 2006, 20:W13-23.

17. Robertson DL, Anderson JP, Bradac JA, Carr JK, Foley B, Funkhouser RK, Gao F, Hahn BH, Kalish ML, Kuiken C, Learn GH, Leitner T, McCutchan F, Osmanov S, Peeters M, Pieniazek D, Salminen M, Sharp PM, Wolinsky S, Korber B: HIV-1 nomenclature proposal. Science 2000, 288:55-56.

18. McCutchan FE: Understanding the genetic diversity of HIV-1. AIDS 2000, 14(Suppl 3):S31-44.

19. Howard TM, Olaylele DO, Rasheed S: Sequence analysis of the glycoprotein 120 coding region of a new HIV type 1 subtype A strain (HIV-1lbNg) from Nigeria. AIDS Res Hum Retroviruses 1994, 10:1755-1757.

20. Carr JK, Torimiro JN, Wolfe ND, Eitel MN, Kim B, Sanders-Buell E, Jagodzinski LL, Gotte D, Burke DS, Birx DL, McCutchan FE: The AG recombinant IbNG and novel strains of group M HIV-1 are common in Cameroon. Virology 2001, 286:168-181.

21. Gao F, Robertson DL, Carruthers CD, Morrison SG, Jian B, Chen Y, BarreSinoussi F, Girard M, Srinivasan A, Abimiku AG, Shaw GM, Sharp PM, Hahn BH: A comprehensive panel of near-full-length clones and reference sequences for non-subtype $B$ isolates of human immunodeficiency virus type 1. J Virol 1998, 72:5680-5698.

22. Heyndrickx L, Janssens W, Zekeng L, Musonda R, Anagonou S, Auwera Van der G, Coppens S, Vereecken K, De Witte K, Van Rampelbergh R, Kahindo M, Morison L, McCutchan FE, Carr JK, Albert J, Essex M, Goudsmit J, Asjo B, Salminen M, Buve A, Groen van Der G: Simplified strategy for detection of recombinant human immunodeficiency virus type 1 group $M$ isolates by gag/env heteroduplex mobility assay. Study Group on Heterogeneity of HIV Epidemics in African Cities. J Virol 2000, 74:363-370.

23. Fonjungo PN, Mpoudi EN, Torimiro JN, Alemnji GA, Eno LT, Nkengasong JN, Gao F, Rayfield M, Folks TM, Pieniazek D, Lal RB: Presence of diverse human immunodeficiency virus type 1 viral variants in Cameroon. AIDS Res Hum Retroviruses 2000, 16:1319-1324.

24. Carr JK, Salminen MO, Albert J, Sanders-Buell E, Gotte D, Birx DL, McCutchan FE: Full genome sequences of human immunodeficiency virus type 1 subtypes $\mathrm{G}$ and $\mathrm{A} / \mathrm{G}$ intersubtype recombinants. Virology 1998, 247:22-31.

25. Abecasis $A B$, Lemey $P$, Vidal $N$, de Oliveira $T$, Peeters $M$, Camacho $R$, Shapiro B, Rambaut A, Vandamme AM: Recombination confounds the early evolutionary history of human immunodeficiency virus type 1 : subtype $\mathrm{G}$ is a circulating recombinant form. J Virol 2007, 81:8543-8551.

26. Piyasirisilp S, McCutchan FE, Carr JK, Sanders-Buell E, Liu W, Chen J, Wagner R, Wolf H, Shao Y, Lai S, Beyrer C, Yu XF: A recent outbreak of human immunodeficiency virus type 1 infection in southern China was initiated by two highly homogeneous, geographically separated strains, circulating recombinant form $\mathrm{AE}$ and a novel $\mathrm{BC}$ recombinant. J Virol 2000, 74:11286-11295.

27. Su L, Graf M, Zhang $Y$, von Briesen $H$, Xing $H$, Kostler J, Melzl $H$, Wolf $H$, Shao $Y$, Wagner R: Characterization of a virtually full-length human immunodeficiency virus type 1 genome of a prevalent intersubtype (C) B') recombinant strain in China. J Virol 2000, 74:11367-11376.

28. Yang R, Xia X, Kusagawa S, Zhang C, Ben K, Takebe Y: On-going generation of multiple forms of HIV-1 intersubtype recombinants in the Yunnan Province of China. Aids 2002, 16:1401-1407.

29. Yu XF, Liu W, Chen J, Kong W, Liu B, Yang J, Liang F, McCutchan F, Piyasirisilp S, Lai S: Rapid dissemination of a novel B/C recombinant HIV-1 among injection drug users in southern China. AIDS 2001, 15:523-525.

30. Luo CC, Tian C, Hu DJ, Kai M, Dondero T, Zheng X: HIV-1 subtype $C$ in China. Lancet 1995, 345:1051-1052.

31. Beyrer C, Razak MH, Lisam K, Chen J, Lui W, Yu XF: Overland heroin trafficking routes and HIV-1 spread in south and south-east Asia. Aids 2000, 14:75-83.

32. Shao $Y Z Y$, Chen $Z$, et al: Isolation of viruses from HIV infected individuals in Yunnan. Chin J Epidemiol 1991, 12:129.

33. Shao YZQ, Wang B, et al: Sequence analysis of HIV env ene among HIV infected IDUs in Yunnan epidemic area of China. Chin J Virol 1994, 10:291-299.
34. Tee KK, Pybus OG, Li XJ, Han X, Shang H, Kamarulzaman A, Takebe Y: Temporal and spatial dynamics of human immunodeficiency virus type 1 circulating recombinant forms 08 BC and 07_BC in Asia. J Virol 2008, 82:9206-9215.

35. Takebe Y, Motomura K, Tatsumi M, Lwin HH, Zaw M, Kusagawa S: High prevalence of diverse forms of HIV-1 intersubtype recombinants in Central Myanmar: geographical hot spot of extensive recombination. AIDS 2003, 17:2077-2087.

36. Chu TX, Levy JA: Injection drug use and HIV/AIDS transmission in China. Cell Res 2005, 15:865-869.

37. Laeyendecker O, Zhang GW, Quinn TC, Garten R, Ray SC, Lai S, Liu W, Chen J, Yu XF: Molecular epidemiology of HIV-1 subtypes in southern China. J Acquir Immune Defic Syndr 2005, 38:356-362.

38. Chang SY, Sheng WH, Lee CN, Sun HY, Kao CL, Chang SF, Liu WC, Yang JY, Wong WW, Hung CC, Chang SC: Molecular epidemiology of HIV type 1 subtypes in Taiwan: outbreak of HIV type 1 CRF07_BC infection in intravenous drug users. AIDS Res Hum Retroviruses 2006, 22:1055-1066.

39. Lim WL, Xing $\mathrm{H}$, Wong KH, Wong MC, Shao YM, Ng MH, Lee SS: The lack of epidemiological link between the HIV type 1 infections in Hong Kong and Mainland China. AIDS Res Hum Retroviruses 2004, 20:259-262.

40. De Sa Filho DJ, Sucupira MC, Caseiro MM, Sabino EC, Diaz RS, Janini LM: Identification of two HIV type 1 circulating recombinant forms in Brazil. AIDS Res Hum Retroviruses 2006, 22:1-13.

41. Aulicino PC, Kopka J, Mangano AM, Rocco C, lacono M, Bologna R, Sen L: Circulation of novel HIV type $1 \mathrm{~A}, \mathrm{~B} / \mathrm{C}$, and $\mathrm{F}$ subtypes in Argentina. AIDS Res Hum Retroviruses 2005, 21:158-164.

42. Rainwater S, DeVange S, Sagar M, Ndinya-Achola J, Mandaliya K, Kreiss JK, Overbaugh J: No evidence for rapid subtype $C$ spread within an epidemic in which multiple subtypes and intersubtype recombinants circulate. AIDS Res Hum Retroviruses 2005, 21:1060-1065.

43. Carr JK, Avila M, Gomez Carrillo M, Salomon H, Hierholzer J, Watanaveeradej V, Pando MA, Negrete M, Russell KL, Sanchez J, Birx DL, Andrade R, Vinoles J, McCutchan FE: Diverse BF recombinants have spread widely since the introduction of HIV-1 into South America. Aids 2001, 15:F41-47.

44. Bartolo I, Rocha C, Bartolomeu J, Gama A, Marcelino R, Fonseca M, Mendes A, Epalanga M, Silva PC, Taveira N: Highly divergent subtypes and new recombinant forms prevail in the HIV/AIDS epidemic in Angola: new insights into the origins of the AIDS pandemic. Infect Genet Evol 2009, 9:672-682.

45. Fontella R, Soares MA, Schrago CG: On the origin of HIV-1 subtype C in South America. AIDS 2008, 22:2001-2011.

46. Bello G, Passaes CP, Guimaraes ML, Lorete RS, Matos Almeida SE, Medeiros RM, Alencastro PR, Morgado MG: Origin and evolutionary history of HIV-1 subtype C in Brazil. AIDS 2008, 22:1993-2000.

47. Sierra M, Thomson MM, Rios M, Casado G, Castro RO, Delgado E, Echevarria G, Munoz M, Colomina J, Carmona R, Vega Y, Parga EV, Medrano L, Perez-Alvarez L, Contreras G, Najera R: The analysis of near fulllength genome sequences of human immunodeficiency virus type $1 \mathrm{BF}$ intersubtype recombinant viruses from Chile, Venezuela and Spain reveals their relationship to diverse lineages of recombinant viruses related to CRF12 BF. Infect Genet Evol 2005, 5:209-217.

48. Thomson MM, Delgado E, Herrero I, Villahermosa ML, Vazquez-de Parga E, Cuevas MT, Carmona R, Medrano L, Perez-Alvarez L, Cuevas L, Najera R: Diversity of mosaic structures and common ancestry of human immunodeficiency virus type $1 \mathrm{BF}$ intersubtype recombinant viruses from Argentina revealed by analysis of near full-length genome sequences. J Gen Virol 2002, 83:107-119.

49. Gomez-Carrillo M, Pampuro S, Duran A, Losso M, Harris DR, Read JS, Duarte G, De Souza R, Soto-Ramirez L, Salomon H: Analysis of HIV type 1 diversity in pregnant women from four Latin American and Caribbean countries. AIDS Res Hum Retroviruses 2006, 22:1186-1191.

50. de Souza AC, de Oliveira CM, Rodrigues CL, Silva SA, Levi JE: Short communication: Molecular characterization of HIV type $1 \mathrm{BF}$ pol recombinants from Sao Paulo, Brazil. AIDS Res Hum Retroviruses 2008, 24:1521-1525.

51. Aulicino PC, Bello G, Rocco C, Romero H, Mangano A, Morgado MG, Sen L: Description of the first full-length HIV type 1 subtype F1 strain in Argentina: implications for the origin and dispersion of this subtype in South America. AIDS Res Hum Retroviruses 2007, 23:1176-1182. 
52. Murphy E, Korber B, Georges-Courbot MC, You B, Pinter A, Cook D, Kieny MP, Georges A, Mathiot C, Barre-Sinoussi F, et al: Diversity of V3 region sequences of human immunodeficiency viruses type 1 from the central African Republic. AIDS Res Hum Retroviruses 1993, 9:997-1006.

53. Carr JK, Salminen MO, Koch C, Gotte D, Artenstein AW, Hegerich PA, St Louis D, Burke DS, McCutchan FE: Full-length sequence and mosaic structure of a human immunodeficiency virus type 1 isolate from Thailand. J Virol 1996, 70:5935-5943.

54. Njai HF, Gali Y, Vanham G, Clybergh C, Jennes W, Vidal N, Butel C, MpoudiNgolle E, Peeters M, Arien KK: The predominance of Human Immunodeficiency Virus type 1 (HIV-1) circulating recombinant form 02 (CRF02_AG) in West Central Africa may be related to its replicative fitness. Retrovirology 2006, 3:40.

55. Konings FA, Burda ST, Urbanski MM, Zhong P, Nadas A, Nyambi PN: Human immunodeficiency virus type 1 (HIV-1) circulating recombinant form 02_AG (CRF02_AG) has a higher in vitro replicative capacity than its parental subtypes A and G. J Med Virol 2006, 78:523-534.

56. McCutchan FE: Global epidemiology of HIV. J Med Virol 2006, 78(Suppl 1): S7-S12.

57. Powell RL, Zhao J, Konings FA, Tang S, Nanfack A, Burda S, Urbanski MM, Saa DR, Hewlett I, Nyambi PN: Identification of a novel circulating recombinant form (CRF) 36_cpx in Cameroon that combines two CRFs (01_AE and 02_AG) with ancestral lineages of subtypes A and G. AIDS Res Hum Retroviruses 2007, 23:1008-1019.

58. Yang R, Kusagawa S, Zhang C, Xia X, Ben K, Takebe Y: Identification and characterization of a new class of human immunodeficiency virus type 1 recombinants comprised of two circulating recombinant forms, CRF07_BC and CRF08_BC, in China. J Virol 2003, 77:685-695.

59. Qiu Z, Xing H, Wei M, Duan Y, Zhao Q, Xu J, Shao Y: Characterization of five nearly full-length genomes of early HIV type 1 strains in Ruili city: implications for the genesis of CRF07_BC and CRF08_BC circulating in China. AIDS Res Hum Retroviruses 2005, 21:1051-1056.

60. Martins Lde O, Leal E, Kishino H: Phylogenetic detection of recombination with a Bayesian prior on the distance between trees. PLOS ONE 2008, 3: e2651.

61. Bello G, Guimaraes ML, Morgado MG: Evolutionary history of HIV-1 subtype B and F infections in Brazil. Aids 2006, 20:763-768.

62. Bello G, Eyer-Silva WA, Couto-Fernandez JC, Guimaraes ML, ChequerFernandez SL, Teixeira SL, Morgado MG: Demographic history of HIV-1 subtypes B and F in Brazil. Infect Genet Evol 2007, 7:263-270.

63. Kiwanuka N, Laeyendecker O, Robb M, Kigozi G, Arroyo M, McCutchan F, Eller LA, Eller M, Makumbi F, Birx D, Wabwire-Mangen F, Serwadda D, Sewankambo NK, Quinn TC, Wawer M, Gray R: Effect of human immunodeficiency virus Type 1 (HIV-1) subtype on disease progression in persons from Rakai, Uganda, with incident HIV-1 infection. J Infect Dis 2008, 197:707-713.

64. A Hall T: BioEdit: a user-friendly biological sequence alignment editor and analysis program for Windows 95/98/NT. Nucl Acids Symp Ser 1999, 41:95-98.

65. Zhang M, Schultz AK, Calef C, Kuiken C, Leitner T, Korber B, Morgenstern B, Stanke M: jpHMM at GOBICS: a web server to detect genomic recombinations in HIV-1. Nucleic Acids Res 2006, 34:W463-465.

66. Schultz AK, Zhang M, Leitner T, Kuiken C, Korber B, Morgenstern B, Stanke M: A jumping profile Hidden Markov Model and applications to recombination sites in HIV and HCV genomes. BMC Bioinformatics 2006, 7:265.

67. Swofford D: PAUP: phylogenetic analysis using parsimony, version 3.1. 1991.

68. Guindon S, Gascuel O: A simple, fast, and accurate algorithm to estimate large phylogenies by maximum likelihood. Syst Biol 2003, 52:696-704.

69. Hillis DM, Bull JJ: An empirical test of bootstrapping as a method for assessing confidence in phylogenetic trees. Syst Biol 1993, 42:182-192.

70. Gaschen B, Taylor J, Yusim K, Foley B, Gao F, Lang D, Novitsky V, Haynes B, Hahn BH, Bhattacharya T, Korber B: Diversity considerations in HIV-1 vaccine selection. Science 2002, 296:2354-2360.

doi:10.1186/1742-4690-7-25

Cite this article as: Zhang et al:: The role of recombination in the emergence of a complex and dynamic HIV epidemic. Retrovirology 2010 7:25.

\section{Submit your next manuscript to BioMed Central and take full advantage of:}

- Convenient online submission

- Thorough peer review

- No space constraints or color figure charges

- Immediate publication on acceptance

- Inclusion in PubMed, CAS, Scopus and Google Scholar

- Research which is freely available for redistribution 